\title{
Récents développements en technologie de radioprotection aux Etats-Unis
}

\author{
C.J. WOOD *
}

(Manuscrit reçu le 24 novembre 1989)

\begin{abstract}
RÉSUMÉ La mise en ceuvre de technologies permettant de contrôler les champs de rayonnement dans les centrales nucléaires américaines s'effectue à un rythme soutenu et a permis à certains exploitants de réduire la radioexposition professionnelle au cours de ces dernières années. Des études récentes visant à remplacer le cobalt, à développer des programmes d'injection de zinc dans les réacteurs à eau bouillante et des programmes de chimle à pH élevé pour les réacteurs REP, à démontrer des méthodes de préconditionnement pour le remplacement des composants et à qualifier la décontamination chimique, ont fourni aux explojtants des méthodes optimisées permettant de réduire la radioexposition.
\end{abstract}

ABSTRACT The implementation of technology to control radiation fields at U.S. nuclear power plants is occurring at a rapid pace and has helped utilities achieve significant reductions in occupational exposures in recent years. Recent studies to replace cobalt, develop BWR zinc injection and PWR elevated pH chemistry programs, demonstrate preconditioning methods for replacement of components, and qualify chemical decontamination have provided utilities with cost-effective methods for minimizing exposures.

\section{TENDANCES DE LA RADIOEXPOSITION}

Les chiffres de radioexposition professionnelle décroissent chaque année depuis 1983. La figure 1 montre que la production totale d'électricité dans les centrales américaines a augmenté de $45,8 \%$ entre 85 et 88 , alors que la dose collective (en homme-rem) a décru de $8 \%$. Pendant ces trois années, 20 nouvelles centrales ont été intégrées à la base de données, le total atteignant 97 en 1988. Dix autres centrales étaient en exploitation mais n'avaient pas accompli le cycle d'une année d'exploitation commerciale entière à la fin 88 .

* Electric power research institute (EPRI), Nuclear power division, 3412 Hillview Avenue, Palo Alto, CA 94303, USA. 


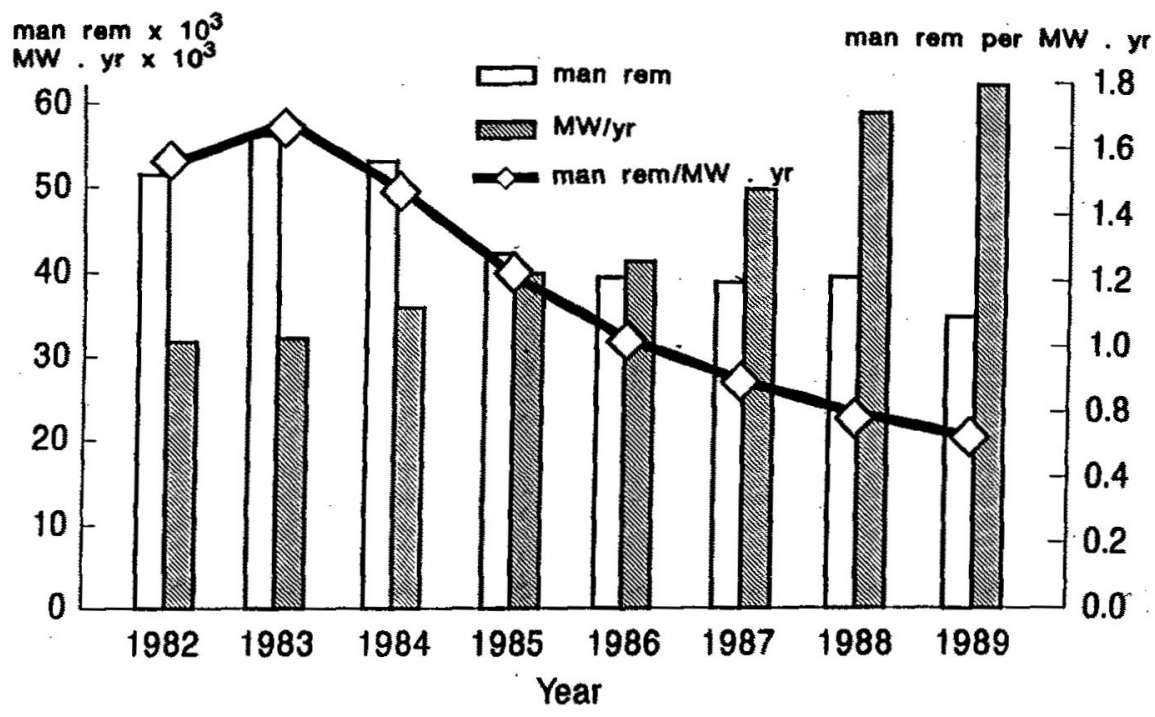

Fig. 1. - Radioexposition et puissance générée par les centrales nucléaires américaines (en rems par an).

La valeur en hommes-rems nécessaire pour produire $1 \mathrm{MW}$-an d'énergie électrique a baissé de $32 \%$ pour les réacteurs REP et de $57,5 \%$ pour les réacteurs à eau bouillante pendant cette même période de trois ans. Le taux global pour les réacteurs à eau légère est à présent de 0,6 homme-rem par MW-an, c'est-à-dire moins de $50 \%$ de la valeur maximale atteinte au début des années 1980 (fig. 1).

Les nouvelles centrales de la base de données sont en partie responsables de cette baisse de la radioexposition. Toutefois, les expositions dans les centrales plus anciennes baissent également. Prenons, par exemple, les 45 centrales REP et les 23 centrales à eau bouillante entrées en service avant 1980. Pour ces deux types de centrales, la comparaison de l'exposition en 1988 et la valeur moyenne entre 1980 et 1983 montre une réduction de 43 et $50 \%$ respectivement (fig. 2). Ces dernières années, le nombre de travailleurs recevant des doses supérieures à $1 \mathrm{rem} / \mathrm{an}$ s'est également réduit de façon significative (fig. 3). En dépit des progrès remarquables en matière de réduction des expositions, la valeur enregistrée dans les centrales américaines est toujours supérieure à celle d'autres nations industrielles disposant de programmes nucléaires importants, mais l'écart se réduit [6]. 
RÉCENTS DÉVELOPPEMENTS EN TECHNOLOGIE DE RADIOPROTECTION AUX ÉTATS-UNIS

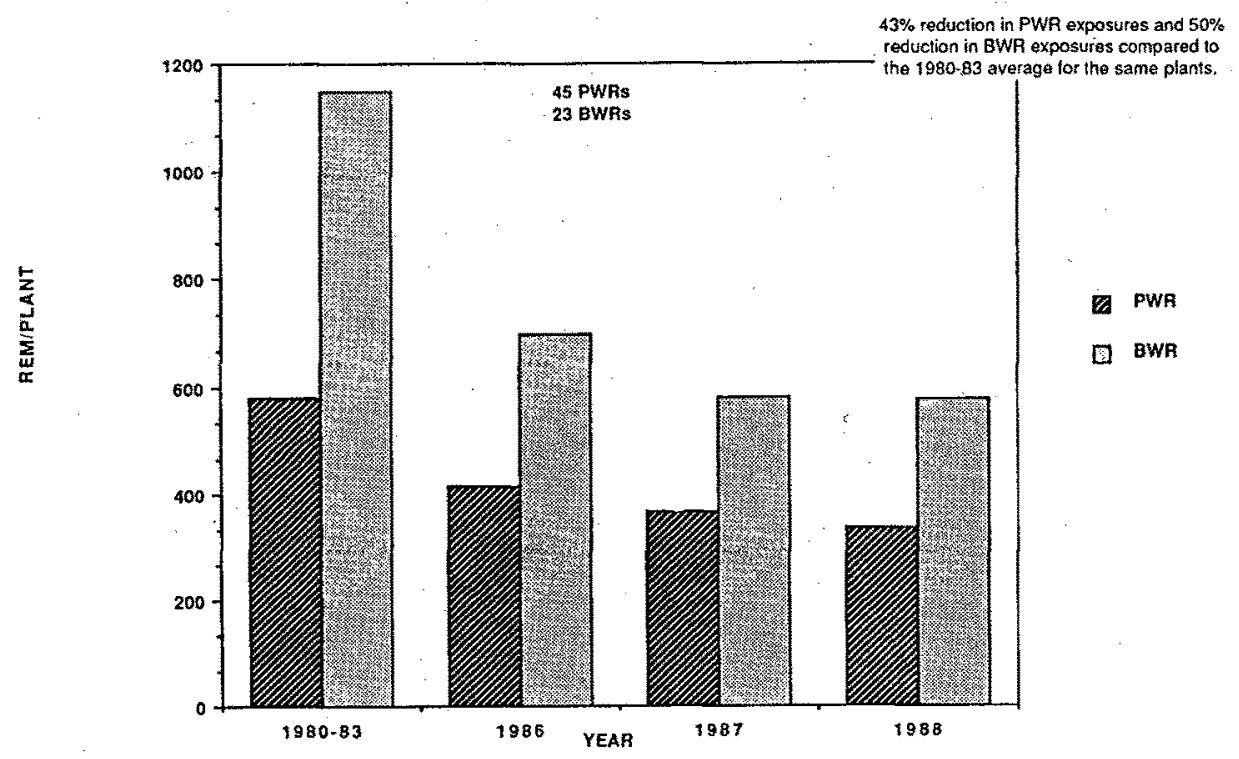

Fig. 2. - Radioexposition dans les centrales en exploitation avant 1980.

$43 \%$ de réduction des expositions pour les REP et $50 \%$ de réduction pour les réacteurs à eau bouillante par rapport à la moyenne obtenue de 1980 à 1983

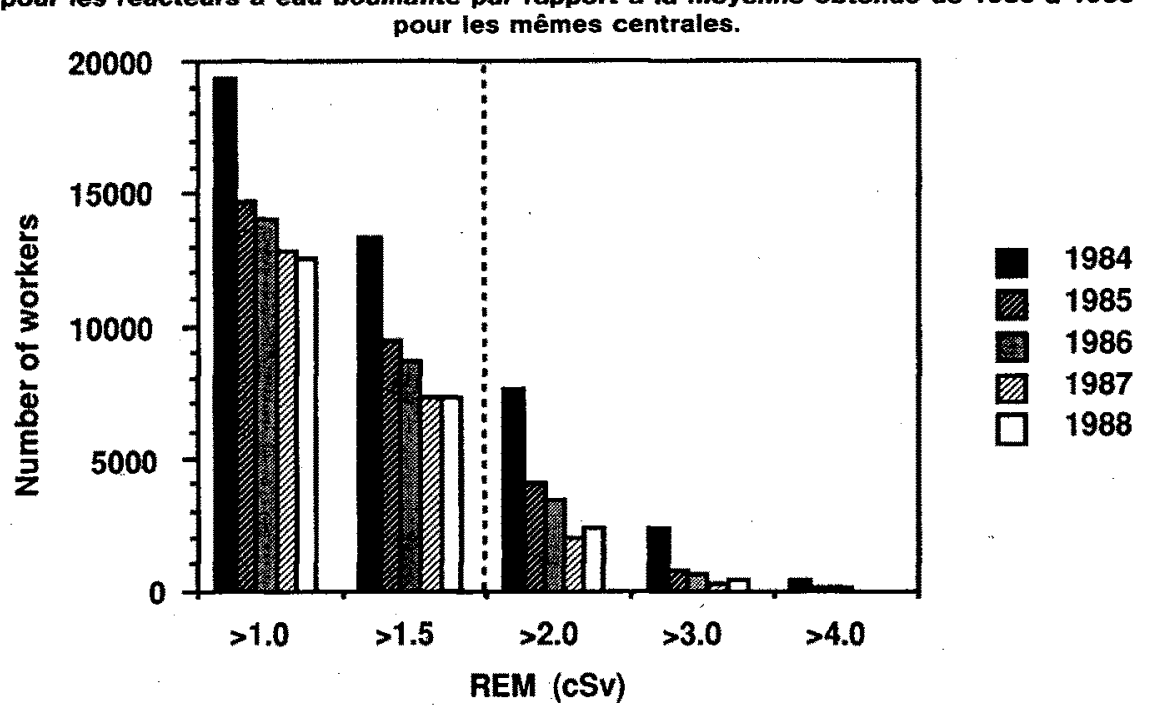

Fig. 3. - Répartition de l'exposition des travalileurs dans les réacteurs à eau légère. 
Le succès de l'industrie nucléaire en matière de réduction des expositions peut être attribué à trois facteurs :

1. amélioration des performances des centrales, avec une réduction des conditions de maintenance, de réparation et de modifications d'équipement ;

2. augmentation de la productivité des agents en zone sous rayonnement, résultat de l'amélioration des procédures de radioprotection ;

3. utilisation de technologies évoluées permettant de réduire les champs de rayonnement et utilisation de dispositifs automatisés.

L'importance de ces trois facteurs varie considérablement d'une centrale à l'autre, mais, globalement, ces trois catégories ont probablement apporté, chacune, une contribution égale au cours de ces dernières années.

La figure 2 montre que le rythme d'amélioration ralentit, peut-être parce que les avantages principaux tirés des facteurs 1 et 2 ont déjà été exploités. Toutefois, les gains potentiels offerts par le facteur 3 restent à mettre en cuvre. A l'avenir, il sera encore plus important de réduire les champs de rayonnement si la tendance à la baisse en matière de radioexposition doit se poursuivre et cette réduction sera particulièrement importante pour contrôler l'exposition des travailleurs occupant des postes clés.

\section{TECHNOLOGIE DE CONTRÔLE DES CHAMPS DE RAYONNEMENT}

Le contrôle des champs de rayonnement couvre quatre domaines, correspondant aux processus fondamentaux liés à l'activation, au transport et au dépôt des produits de corrosion et d'usure contenant du cobalt et se trouvant dans les systèmes primaires [3]. Les programmes de contrôle les plus performants comportent au moins trois des quatre méthodes, que l'on décrira ci-dessous.

\section{Réduire la source}

La réduction de la source implique la réduction du niveau d'impureté du cobalt dans les alliages structurels utilisés pour fabriquer les composants du réacteur et le remplacement des alliages d'apport à base de cobalt par des alliages d'apport sans cobalt, les alliages à base de cobalt étant généralement utilisés dans la robinetterie nucléaire et dans d'autres composants nécessitant une résistance supérieure à l'usure.

L'utilisation de tube en inconel 690 à faible teneur en cobalt vient d'être recommandée pour les générateurs de vapeur de remplacement. L'utilisation de grilles en zircaloy et non plus en inconel dans les ensembles combustible REP permet de réduire considérablement les inventaires de cobalt 60 . Des alliages remplaçant les alliages à base 
de cobalt sont actuellement utilisés avec succès sur les aiguilles et rouleaux des lames de contrôle des réacteurs à eau bouillante ainsi que dans les soupapes de régulation de débit. Une étude placée sous l'égide de l'EPRI a permis de développer des alliages d'apport à base de fer résistant à l'usure et baptisés NOREM [4]. Cet alliage, ainsi que deux autres à base de fer, ont été déposés sur les valves à opercule actuellement soumises à des tests d'endurance de longue durée. D'autres travaux actuellement en cours portent sur le développement de procédures de réparation pour ces nouveaux alliages. Par ailleurs, des instructions sont en cours de développement, visant à aider les exploitants nucléaires à mettre en œuvre ces nouveaux alliages et à identifier les types de vannes et les systèmes pour lesquels l'utilisation d'alliages à base de cobalt n'est pas nécessaire [2].

\section{Contrôle du transport et de l'activation}

Un bon programme de chimie de l'eau constitue la solution pour réduire la formation et le dégagement de produits de corrosion dans l'eau du réacteur. II est à noter qu'il existe une importante corrélation entre labsence de points chauds de rayonnement dans les pièges à dépôt des réacteurs à eau bouillante et un bon programme de chimie de l'eau. Une injection de zinc a été effectuée dans cinq réacteurs à eau bouillante aux Etats-Unis depuis que l'on a découvert que la présence de concentration de zinc en parties par milliard dans l'eau des réacteurs à eau bouillante réduit le dégagement de produits de corrosion solubles et empêche ensuite le dépôt de cobalt 60 . La formation de champs de rayonnement est réduite d'environ $50 \%$, et des expériences récentes ont montré que les problèmes en matière d'activité de zinc 65 ont été surmontés [7]. Des études sont actuellement en cours pour déterminer la faisabilité de l'injection de zinc dans les REP.

Dans le cas des REP, le contrôle du pH est extrêmement important pour éviter l'activation des produits de corrosion dus au dépôt à l'intérieur du cour. Les champs de rayonnement dans les générateurs de vapeur des centrales utilisant un $\mathrm{pH}$ élevé et bien contrôlé sont de 1,5 à 3 fois inférieurs à ceux des centrales disposant d'une chimie classique. Le but des recherches actuelles est de déterminer jusqu'à quel point on peut augmenter le $\mathrm{pH}$ sans porter atteinte à l'intégrité du gainage du combustible et des tuyauteries du générateur de vapeur. La centrale de Millstone-3 a mis en cuvre un programme d'augmentation du $\mathrm{pH}$ utilisant $3,5 \mathrm{ppm}$ de lithium. Les principales conclusions tirées du premier cycle d'exploitation avec augmentation du lithium (second cycle de cette centrale) sont à présent disponibles. Les champs de rayonnement des tuyauteries du système primaire ont augmenté en moyenne de $11 \%$ et ceux des boîtes à eau baissé de $3 \%$ par rapport au premier cycle d'exploitation. En général, à cette étape de la vie d'une centrale, on s'attend à une augmentation de 25 à $30 \%$. La figure 4 propose une comparaison entre les données de la centrale de Millstone- 3 et les valeurs supérieures, moyennes et inférieures relevées sur les centrales américaines de Westinghouse. La centrale de Millstone-3 se situe au bas de la plage inférieure [1]. 


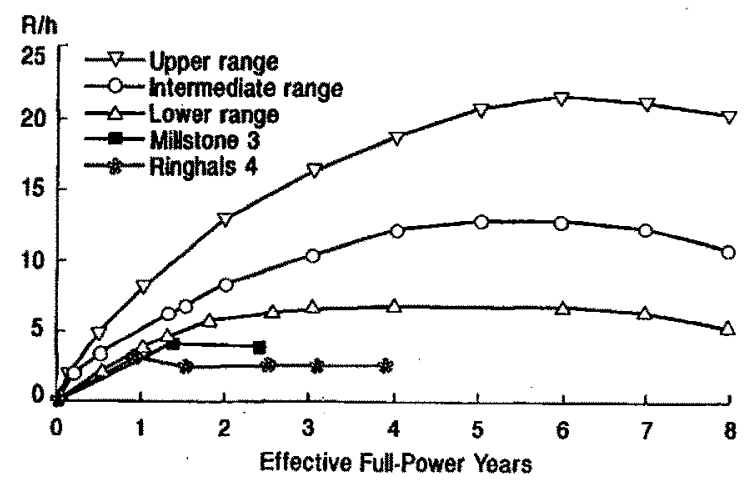

Fig. 4. - Champs de rayonnement des générateurs de vapeur dans les réacteurs REP Westinghouse.

\section{Contrôle des dépôts à l'extérieur du cceur}

Le préconditionnement des surfaces du système primaire, soit avant le démarrage de la centrale, soit avant l'installation des composants de remplacement, permet de réduire considérablement le taux de dépôt des produits de corrosion activés. Il existe deux méthodes principales de préconditionnement : modification en surface par polissage mécanique ou électrochimique et préoxydation chimique permettant de développer une pellicule d'oxyde passive avant d'exposer la surface au liquide réfrigérant du réacteur. L'association de la méthode d'électropolissage et de préoxydation par air humide permet d'obtenir les taux les plus faibles de recontamination sur les systèmes des tuyauteries de recirculation de remplacement dans les réacteurs à eau boullante. Tous les remplacements de tuyauteries effectués récemment ont utilisé des tuyauteries polies électrolytiquement, la majorité ayant également été préoxydée.

Des essais portant sur le polissage mécanique et électrochimique effectués à la centrale de Chinon $\mathrm{B}-2$ ont montré que le dépôt de cobalt sur l'acier inoxydable des générateurs de vapeur peut être utilisé avec un facteur de 4 à 10 [5]. A partir de cette expérience concluante, l'EPRI a mis en place des programmes de développement et de qualification visant à appliquer les techniques de modification de surface des boîtes à eau de générateurs de vapeur REP nouvelles ou de remplacement. Ce programme, qui vient d'être achevé, a fourni des données montrant que le polissage électrolytique constitue une technique acceptable pour les surfaces des générateurs de vapeur des réacteurs REP. L'intégrité métallurgique du recouvrement de soudure n'a pas été affectée, et ce, dans la large gamme des paramètres de polissage électrolytique. Plusieurs exploitants américains envisagent actuellement le conditionnement de surface des boîtes à eau des générateurs de vapeur REP. 


\section{Décontamination}

L'activité déposée peut être éliminée par décontamination mécanique ou chimique. La contamination mécanique est utilisée pour les composants ou les surfaces de taille réduite tandis que la décontamination chimique est destinée au système de refroidissement du réacteur. Pour les centrales présentant des champs de rayonnement élevés, la décontamination chimique partielle des systèmes s'est avérée extrêmement rentable. Dans la majorité des réacteurs à eau bouillante anciens, les systèmes de tuyauterie ont été à présent décontaminés au moins une fois, certains ayant subi cette opération trois fois. La technique LOMI a été la plus couramment utilisée pendant les trois dernières années. Les opérations de décontamination des générateurs de vapeur ont été moins nombreuses, du fait de leur coût élevé dû à l'utilisation de bouchons de tubulure [8].

Une récente étude de faisabilité a montré que la décontamination d'un système entier est plus efficace que la décontamination partielle de système actuellement utilisée et qu'elle permettra d'exécuter la plupart des travaux en réduisant la radioexposition. Cette étude conclut que la décontamination globale est rentable pour les réacteurs à eau bouillante et à eau sous pression, à condition qu'elle ait lieu lors d'un arrêt de tranche important durant lequel d'autres tâches d'inspection, de réparation ou de remplacement seraient effectuées. Une étude récente a été mise en place auprès des fabricants de chaudières nucléaires à propos d'autres problèmes techniques et de licence. On espère que les résultats de cette étude encourageront un exploitant américain à effectuer une décontamination globale après retrait du combustible au début des années 90.

\section{CONCLUSIONS}

La combinaison des programmes de réduction du cobalt, d'amélioration de la chimie de l'eau, de décontamination et de modification en surface des composants de remplacement permet de réduire considérablement les champs de rayonnement dans les centrales ayant déjà quelques années d'expérience. L'efficacité des techniques de contrôle de radiation est encore plus importante lorsqu'elles sont appliquées dès la mise en service, comme c'est le cas dans la majorité des centrales EDF. Dans ce cas, l'absence de cobalt dans le cœur et un bon contrôle de la chimie ont permis de réduire les champs de rayonnement. L'application des technologies de décontamination et de préconditionnement des surfaces facilitera le contrôle des expositions avant et après le remplacement des générateurs de vapeur. 


\section{RËFÉRENCES}

[1] BERGMANN C.A., JACKO R., ROESMER J., MILLLER R., HUDSON M.J.B., BURNS T.F., WOOD C.J. - PWR primary system chemistry : experience with elevated $\mathrm{pH}$ at Millstone Point unit 3 . In : Water chemistry of nuclear reactor systems 5. London : British nuclear energy society (BNES) 1989, vol. 2, 7-12.

[2] Cobalt reduction guidelines. Palo Alto, CA : Electric power research institute. NP-6737, 1990.

[3] OCKEN H. - Activation of cobalt released by in-core and ex-core sources, Healt Phys. 1990, 58 (3), 259-261.

[4] OCKEN H. - Qualification of cobalt-free hardfacing alloys for dose control. In : Proceedings, international workshop on new developments in occupational dose control. NUREG/CP-0110, 1990.

[5] SAURIN P., WEBER C., BRISSAUD A., GOUILLARDOU G. - French experience with electropolishing. In : Water chemistry of nuclear reactor system 5 . London : British nuclear energy society (BNES), 1989, vol. 2, 23-28.

[6] WOOD C.J. - Radiation exposure trends : closing the gap with Europe, Power 1990.

[7] WOOD C.J., MARBLE W.J., PRYSTUPA M., HUDSON M.J.B., WILKENS D.L. Experience with zinc injection passivation at BWR plants in the USA. In : Water chemistry of nuclear reactor systems 5 . London : British nuclear energy society (BNES), 1989, vol. 1, 111-114.

[8] WOOD C.J. - Progress of the application of chemical decontamination technology in the United States. Progress Nucl. Energy, 1990, $23(1), 35-80$. 